\title{
ОЦЕНКА ДЕФОРМИРУЕМОСТИ МЕТАЛЛА ПРИ ХОЛОДНОМ ФОРМООБРАЗОВАНИИ ВНУТРЕННИХ ШЛИЦЕВЫХ ПОВЕРХНОСТЕЙ
}

Основной задачей технологии металлообрабатывающего производства является изготовление деталей с наименьшими трудозатратами, с наибольшей производительностью и коэффициентом использования металла, причем их качество должно удовлетворять условиям эксплуатации.

Несмотря на развитие безотходных технологий, в частности, методов обработки металлов давлением, все еще остается большим объем заготовок, получаемых обработкой резанием.

Для образования в заготовках шлицевых поверхностей в отверстиях принято использовать технологические процессы протягивания, долбления и горячей штамповки выдавливанием [1-6].

Холодная штамповка методом выдавливания является одним из наиболее передовых и прогрессивных процессов изготовления деталей, который обеспечивает резкое повышение производительности труда, значительное сокращение технологических отходов металла, снижение трудоемкости процессов и себестоимости выпускаемой продукции.

Возможности холодной штамповки выдавливанием весьма широки и разнообразны. Этим методом можно изготовить полуфабрикаты и готовые детали самой различной формы, в том числе и внутренние шлицевые профили, из малоуглеродистых, среднеуглеродистых и малолегированных сталей, а также из подавляющего большинства цветных металлов и сплавов.

Примером такой технологии может быть технологический процесс формирования внутреннего шлицевого соединения в трубных заготовках методом их обжатия матрицами на профильных оправках $[7,8]$.

Учитывая необходимость в получении новых экономически выгодных технологий в Институте сверхтвердых материалов им. В. Н. Бакуля АН Украины в отделе № 20 был разработан технологический процесс формирования внутренних шлицевых поверхностей в глухих отверстиях методом холодного пластического деформирования (ХПД).

Суть процесса заключается в том, что заготовка из стали $20 \mathrm{X}$ (рис. 1) вместе с размещенной внутри профильной (шлицевой) оправкой деформируется (проходит) через специальное приспособление для многоступенчатого редуцирования внутренних шлицевых поверхностей в глухих отверстиях. Направляющая часть, внутренний толкатель и шлицевая оправка спроектированы таким образом, чтобы после завершения процесса деформирования автоматически происходил сброс изделия с профильной оправки.

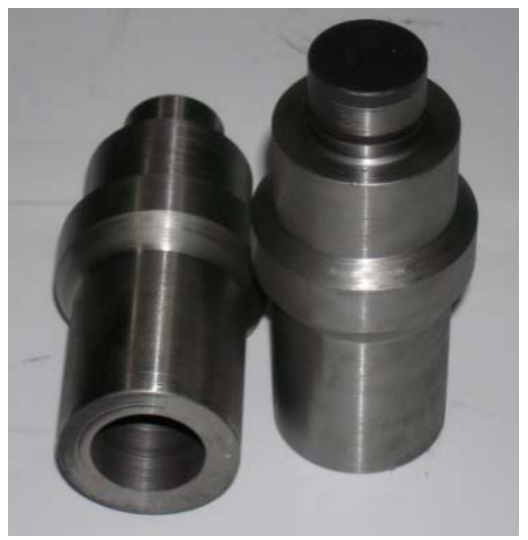

Рис. 1. Заготовка 
Формирование внутренних шлицевых поверхностей в глухих отверстиях методом холодного пластического деформирования обеспечивает качество готовых изделий, используемых в гидротрансмиссии тихоходных машин (рис. 2). Под качеством изделий понимается благоприятное технологическое наследие, а именно: упрочнение металла, остаточные напряжения, микроструктура, а также остаточная пластичность.

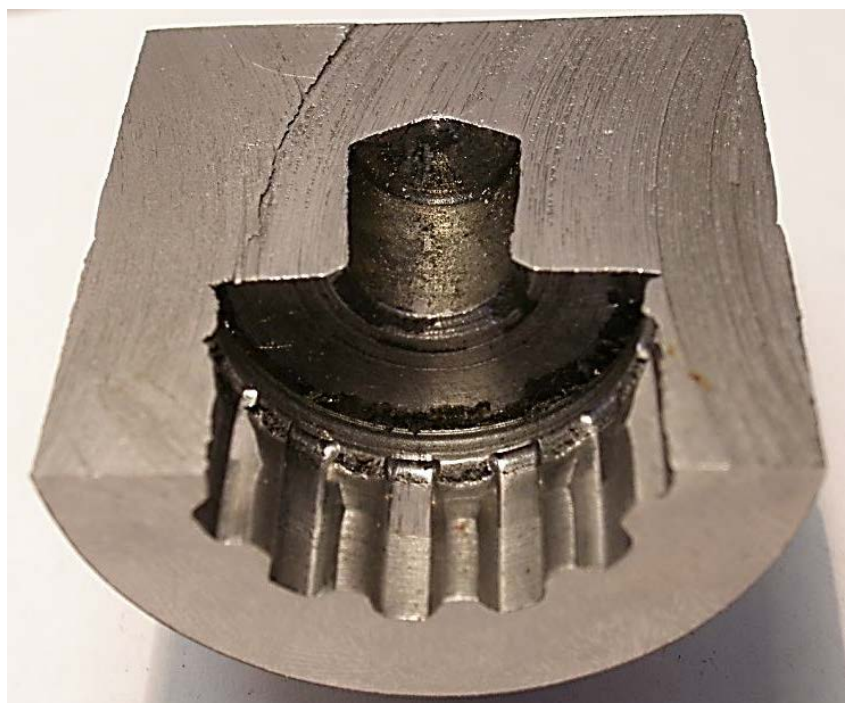

Рис. 2. Деталь с внутренней шлицевой поверхностью в глухом отверстии

Формирование заготовок происходило по различным схемам нагружения (приложения усилия):

- схема № 1 - несвободная оправка - усилие прилагается к хвостовику шлицевой оправки (заг. № 1.1.);

- схема № 2 - комбинированная (заг. № 1.2.);

- схема № 3 - свободная оправка - усилие прилагается к толкателю, давящему на торец заготовки (заг. № 2.1., 2.2., 2.3.).

В данном процессе существует проблема, которая связана с тем, что высота зуба шлица не достигает 10 - 15 \% необходимого заполнения. Кроме того, остается неясным вопрос обеспечения качества шлицевой поверхности с точки зрения несущей способности, а также ресурса пластичности в процессе формирования шлицов.

Поэтому, для усовершенствования данного процесса формирования внутренних шлицевых поверхностей в глухих отверстиях методом холодного пластического деформирования, необходимо разработать рекомендации позволяющие управлять параметрами процесса, обеспечивающими качество изделий.

Целью работы является оценка деформируемости металла при формировании внутренних шлицевых поверхностей в глухих отверстиях методом холодного пластического деформирования. Необходимо определить ресурс пластичности заготовок при различных схемах формирования внутренних шлицевых поверхностей с последующим выбором наиболее благоприятной схемы с точки зрения деформирования заготовки без разрушения.

С целью выяснения возможности использования холодного пластического деформирования без разрушения заготовки в процессе формирования внутренних шлицевых поверхностей исследовали напряженно-деформированное состояние металла (НДС). При этом привлечен метод измерения твердости, предусматривающий построение градуировочных графиков: твердость, интенсивность напряжений, интенсивность деформаций (рис. 3) [8]. Метод твердости позволил учесть упрочнение металла, что дает возможность оценивать напряжение текучести в расчетах НДС. 

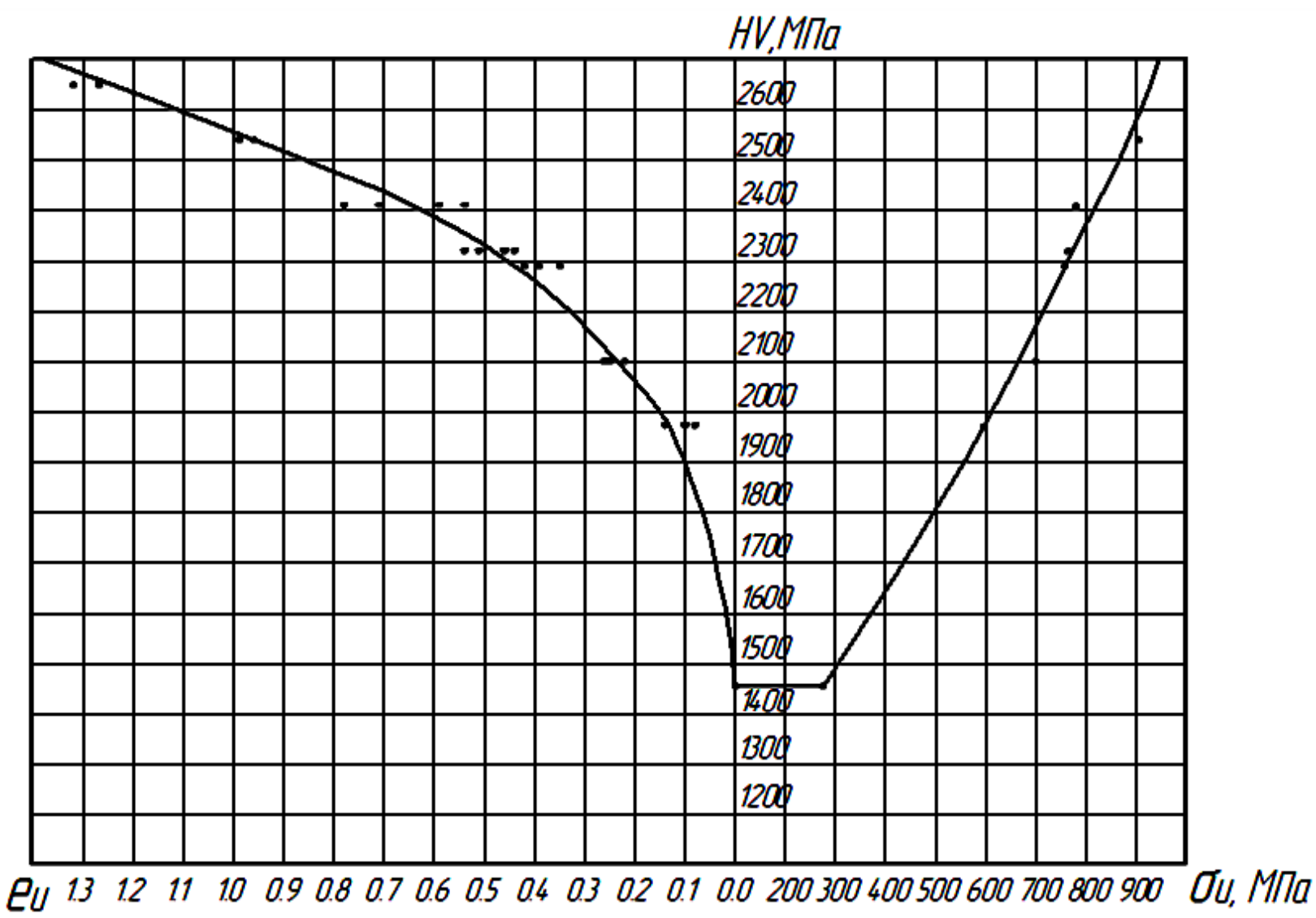

Рис. 3. Градуировочный график стали $20 \mathrm{X}$

(• - эксперимент, - - аппроксимация)

Для изучения распределения твердости, при различных схемах нагружения, вырезанные образцы из цилиндрических шлицевых изделий заливали в специальной обойме эпоксидным клеем, затем по меридиональной плоскости их шлифовали, полировали и измеряли твердость по Виккерсу HV на приборе ТП - 7P - 1 под нагрузкой $50 \mathrm{H}$, выдержкой 12,5 с., как показано на рис. 4 (а, б, в, г, д).

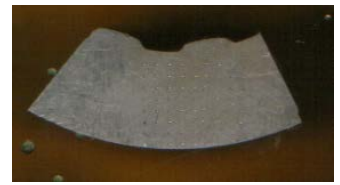

a)

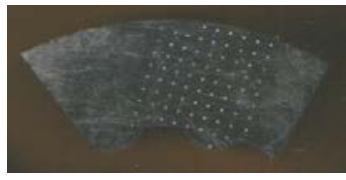

б)

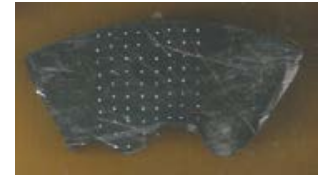

в)

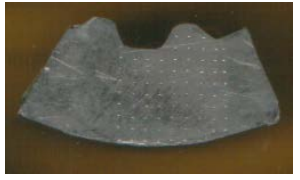

г)

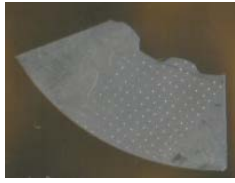

д)

Рис. 4. Измерение твердости на образцах шлицевого профиля при различных схемах нагружения:

a) заг. № 1.1 ; б) заг. № 1.2 ; в) заг. № 2.1 ; г) заг. № 2.2 ; д) заг. № 2.3

После построения графиков распределения твердости строили изолинии (изосклеры) равных твердостей при различных схемах нагружения формирования шлицевого профиля изделия.

Согласно градуировочного графика в каждой точке деформированной области определяли интенсивность напряжений и интенсивность деформаций. 


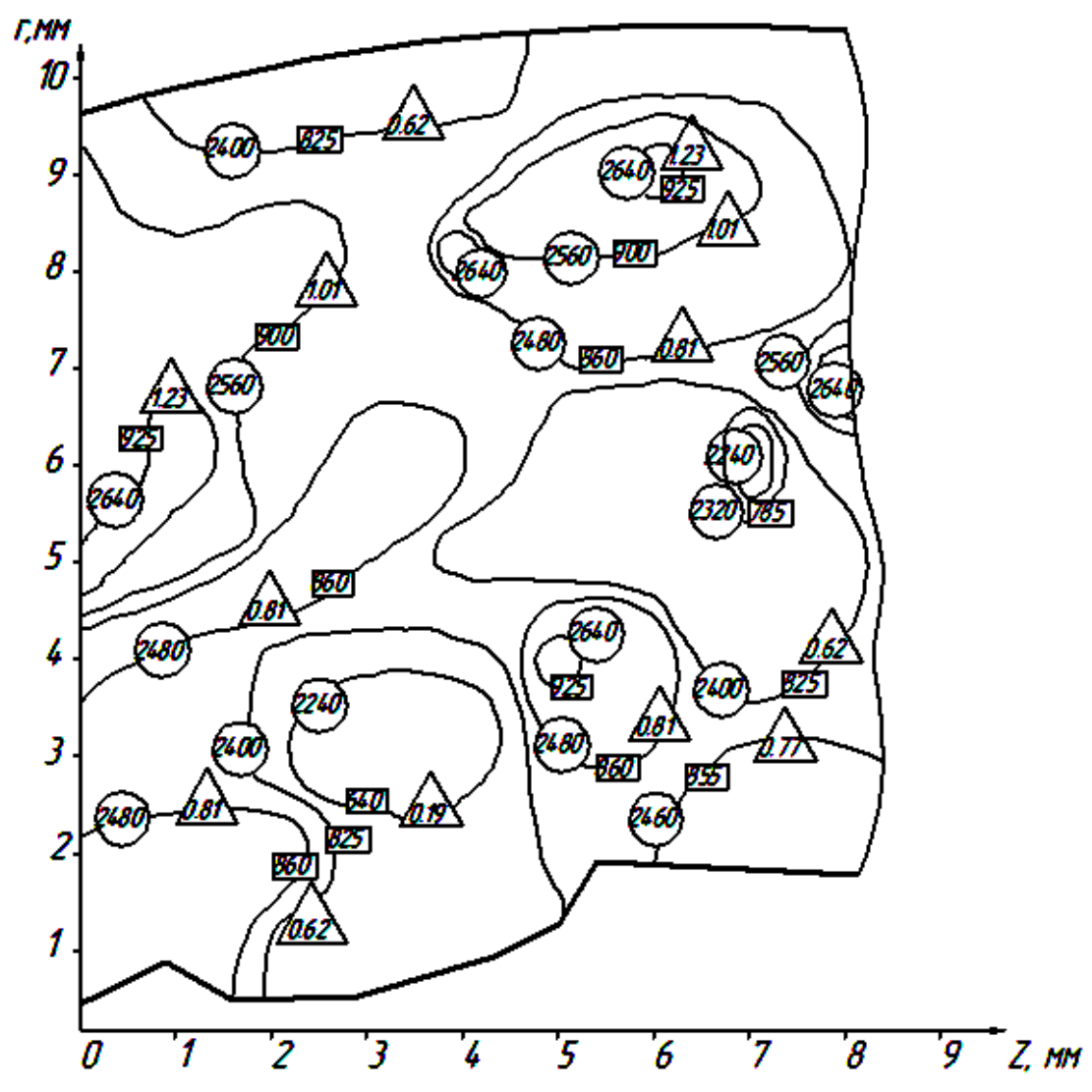

Рис. 5. Изолинии (изосклеры) твердости НV (МПа), интенсивность напряжений $\sigma_{u}($ Ма), интенсивность деформаций - е в меридиональном сечении заготовки № 1.1. с внутренней шлицевой поверхностью

На рис. 5 показано распределение $\mathrm{HV}, \sigma_{\mathrm{u}}$, е $\mathrm{e}_{\mathrm{u}}$ в меридиональном сечении заготовки № 1.1. Как следует из рис. 5 наибольшее значение твердости (HV $=2640$ МПа), а, следовательно, $\sigma_{u}$ и е $\mathrm{u}_{\mathrm{u}}$ наблюдается вблизи центральной части впадины шлицевой поверхности. Наименьшее значение твердости (HV = $2240 \mathrm{MПа)} \mathrm{-} \mathrm{вблизи} \mathrm{зуба} \mathrm{шлицевой} \mathrm{поверхности.}$ В дальнейшем полученные результаты используем для расчета НДС.

Напряженное состояние определяли инженерным методом с привлечением дифференциальных уравнений равновесия и условия пластичности. Использовали методику, изложенную в работах [10-12].

Указанная методика заключается в следующем: при создании математической модели для расчета напряженного состояния процесс получения шлицевого профиля рассматривался как двухэтапный, поскольку одновременно происходит обжатие внешней поверхности втулки матрицей, вдавливание шлицев оправки в материал заготовки и течение металла в межзубовое пространство оправки.

Таким образом, первый этап решения данной задачи был рассмотрен в двух стадиях: внедрение штампа в пластическое полупространство и в полосу конечной толщины.

Зона пластической деформации имеет форму полукруга (рис. 6) с внешним радиусом $\rho_{r}$, определяемым для начального этапа, когда происходит интенсивный рост этой зоны, по эмпирической зависимости:

$$
\rho_{r}=r\left[1,2+2,4 \sqrt{\left(\frac{h}{2 r}+0,5\right)^{2}+0,25}\right]
$$

где $r$ - радиус застойной зоны. 


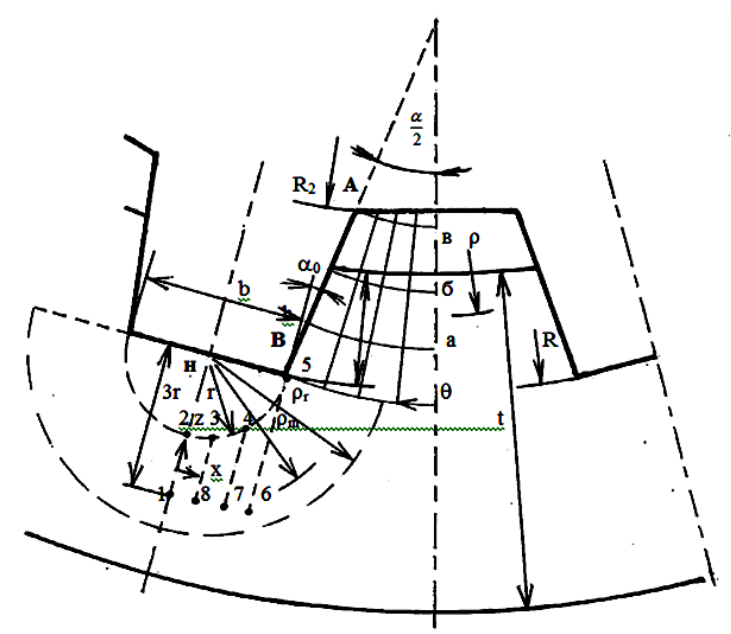

Рис. 6. Расчетная схема для определения напряженно-деформированного состояния при формировании внутреннего шлицевого профиля

При глубине $h \geq d$ значение $\rho_{r} \approx 2,5 d$. В этой же работе приводится зависимость накопленной интенсивности деформации от текущих значений радиуса $\rho_{m}$ в виде:

$$
e_{u}=\ln \frac{e^{\varepsilon_{i}^{\prime}}\left(\rho_{r}^{\prime}-1\right)}{\bar{\rho}\left(e^{\varepsilon_{i}^{\prime}}-2\right)+\bar{\rho}_{r}-e^{\varepsilon_{i}^{\prime}}},
$$

где $\varepsilon_{i}^{\prime}=\frac{h}{r} ; \quad \bar{\rho}_{r}=\frac{\rho_{r}}{r} ; \bar{\rho}=\frac{\rho_{m}}{r}$.

Напряженное состояние определяли поэтапно с использованием инженерного метода, линий скольжения и метода определения напряженно-деформированного состояния по распределению твердости (метода твердости). На первом этапе решали задачу сжатия полосы конечных размеров. Тогда, решая совместно систему уравнений:

$$
\left\{\begin{array}{l}
\frac{d \sigma_{x}}{d x}+\frac{2 \tau_{x z}}{h}=0 \\
\sigma_{x}-\sigma_{z}=\sigma_{u} \\
\tau_{x z}=\mu \sigma_{z}
\end{array}\right.
$$

приходим к решению:

$$
\sigma_{z}=-\sigma_{u} \exp \left[\frac{2 \mu}{h}\left(\frac{b}{2}-x\right)\right],
$$

где $\mathrm{x}$ - расстояние от оси штампа.

Для характерных точек $1 . . .8$ определялось напряженное состояние, расстояние 3r (рис. 6), обусловлено областью распространения пластической деформации. Значение коэффициента трения, согласно табличным данным принимаем $\mu=0,15$. При $t / b<4,37$ принимаем значение коэффициента трения равным $\mu=0,5$.

Первую стадию второго этапа рассматривали как краевую задачу прессования. Введя полярную систему координат и используя гипотезу плоских сечений, совместно решали систему уравнений: 


$$
\left\{\begin{array}{l}
\frac{d \sigma_{\rho}}{d \rho}+\frac{\sigma_{\rho}-\sigma_{\theta}}{\rho}+2 \frac{\tau_{\rho \theta}}{\rho \alpha}=0 \\
\sigma_{\rho}-\sigma_{\theta}=\sigma_{u} \\
\tau_{\rho \theta}=-\mu \sigma_{\theta} \\
\sigma_{u}=A e_{u}^{n}
\end{array}\right.
$$

Из условия постоянства объема материала $e_{\theta}=-e_{\rho}$, тогда интенсивность деформаций определялась как:

$$
e_{u}=1,15 e_{\rho}=1,15 \ln R / \rho .
$$

Во избежание приведения громоздких формул и выводов излагаются только принципы решения на данном этапе:

- при совместном решении уравнения (5) получили неоднородное уравнение первого порядка;

- решение соответствующего однородного уравнения

$$
\frac{d \sigma_{\rho}}{d \rho}-\delta \frac{\sigma_{\rho}}{\rho}=0
$$

будет

$$
\sigma_{\rho}=c \rho^{\delta}
$$

где $\delta=2 \mu / \alpha$.

- неоднородное уравнение решали, используя метод вариации произвольной постоянной в уравнении (8):

$$
\frac{d \sigma_{\rho}}{d \rho}=\frac{d c}{d \rho} \rho^{\delta}+c \delta \rho^{\delta-1}
$$

- подставляя (9) в неоднородное уравнение, получаем:

$$
C=-A \times 1,15^{n}(1+\delta) \int\left(\ln \frac{R}{\rho}\right)^{n} \times \rho^{-1-\delta} d \rho+C_{1},
$$

где $A, n$ - коэффициенты аппроксимации кривой течения (для Ст. $20 \mathrm{X}$ $A=930$ МПа, $n=0,215)$. Принимаем $\sigma_{\rho_{0}}=\sigma_{Z}$, тогда окончательно уравнение для определения $\sigma_{\rho}$ приобретает вид:

$$
\begin{aligned}
& \sigma_{\rho}=-A \times 1,15^{n}(1+\delta) F^{n} R^{k \times n}\left(-\frac{\rho^{-\delta-k \times n}}{k \times n+\delta}\right)+ \\
& +\frac{\sigma_{\rho_{0}}+A \times 1,15^{n}(1+\delta) F^{n} R^{k \times n} \times R^{\delta}\left(-\frac{R^{-\delta-k \times n}}{k \times n+\delta}\right)}{R^{\delta}},
\end{aligned}
$$

где $F$ и $k$ - коэффициенты линеаризации при решении интеграла уравнения (10), где $\ln (R / \rho)$ было принято как $f(R / \rho)$. 
Интенсивность напряжений определяли по последнему соотношению уравнения (5).

Задачу течения металла в сходящемся канале (вторая стадия второго этапа) решали при таких граничных условиях: на оси симметрии при $\theta=0, \tau_{r \theta}=0$, на стенке канала при $\theta=\alpha / 2$ ( $\alpha$ - угол схождения канала), значение $\tau_{r} \theta$ принималось равным найденным ранее значениям при решении краевой задачи. Компоненты тензора напряжений при плоской деформации определяются по соотношениям:

$$
\sigma_{r}=\sigma+k \cos 2 \varphi ; \sigma_{r}=\sigma-k \cos 2 \varphi ; \tau_{r \theta}=k \sin 2 \varphi,
$$

где $\varphi$ - угол между осью $\mathrm{r}$ и направлением главного напряжения $\sigma_{1} ; k-$ интенсивность касательных напряжений, $k=\sigma_{u} / \sqrt{3}$.

Дифференциальные уравнения равновесия имеют вид:

$$
\frac{\partial \sigma_{r}}{\partial r}+\frac{1}{r} \frac{\partial \tau_{r \theta}}{\partial \theta}+\frac{\sigma_{r}-\sigma_{\theta}}{r}=0 ; \frac{\partial \tau_{r \theta}}{\partial r}+\frac{1}{r} \frac{\partial \sigma_{\theta}}{\partial \theta}+\frac{2 \tau_{r \theta}}{r}=0 .
$$

Подставляя в дифференциальное уравнение (13) компоненты тензора напряжений (12), получим уравнение:

$$
\frac{r}{2 k} \frac{\partial \sigma}{\partial r}+\cos 2 \varphi\left(\frac{d \varphi}{d \theta}+1\right)=0 ; \quad \frac{r}{2 k} \frac{\partial \sigma}{\partial \theta}+\sin 2 \varphi\left(\frac{d \varphi}{d \theta}+1\right)=0 .
$$

Поскольку значение $\varphi$ зависит только от $\boldsymbol{\theta}$, то гидростатическое (среднее) напряжение может быть выражено в форме:

$$
\sigma=k\left(1-\varpi(\theta)+2 n \ln \frac{\rho}{R}\right)
$$

где $\varpi(\theta)$ - некоторая функция от $\theta$ и $\varpi(0)=0$.

Подставляя (15) в (14), получим:

$$
\frac{d \varphi}{d \theta}=\frac{n}{\cos 2 \varphi}-1 ; \quad \frac{d \varpi}{d \theta}=n \operatorname{tg} 2 \varphi .
$$

Решение первого из уравнений (16) для положительных значений параметров $n$ - имеет вид:

$$
\theta-\theta_{0}=\frac{n}{\sqrt{n^{2}-1}} \operatorname{arctg}\left(\sqrt{\frac{n+1}{n-1} \operatorname{tg} \varphi}\right)-\varphi
$$

а второго:

$$
2\left(\varpi-\varpi_{0}\right)=n \ln (n-\cos 2 \varphi)
$$

Напряженное состояние сводится к определению постоянных $\theta_{0}, \omega_{0}$, и параметра $n$. Воспользуемся граничными условиями:

1) при $\theta=0 ; \varphi=0 ; \varpi=0$;

2) при $\theta=\alpha / 2$ принимаем $\tau_{r \theta}=k \sin 2 \varphi=-\mu \sigma_{\theta}$. 


$$
\begin{gathered}
\theta=\frac{n}{\sqrt{n^{2}-1}} \operatorname{arctg}\left(\sqrt{\frac{n+1}{n-1} \operatorname{tg} \varphi}\right)-\varphi ; \\
\varpi=\frac{n}{2} \ln \frac{n-\cos 2 \varphi}{n-1} ; \\
\alpha=\frac{2 n}{\sqrt{n^{2}-1}} \operatorname{arctg}\left(\sqrt{\frac{n+1}{n-1} \operatorname{tg} \varphi}\right)-2 \varphi
\end{gathered}
$$

Алгоритм расчета напряжений последней стадии возможен в такой последовательности:

- из граничного условия (19) определяем значение $\varphi$;

- методом минимизации функции (22) определяем параметр $n$;

- используя соотношение (20), находим $\varphi$ для каждого значения $\boldsymbol{\theta}$;

- по соотношению (21) находим значение $\varpi$, затем по (15) находим $\sigma$;

- находим значение компонентов тензора напряжений на линии в (рис. 3.16).

Результаты расчетов напряжений в наиболее характерных точках и областях при формировании внутреннего шлицевого профиля в глухом отверстии приведены в таблице 1.

Расчеты получены с помощью программы MathCAD.

Таблица 1

\begin{tabular}{|c|c|c|c|c|c|c|c|c|}
\hline \multicolumn{9}{|c|}{ Этапы формирования изделия № 1.1} \\
\hline \multicolumn{9}{|c|}{1 этап } \\
\hline $\begin{array}{c}\text { Параметры, } \\
\text { мм }\end{array}$ & Точки & $\begin{array}{l}\mathrm{HV}, \\
\mathrm{M} \Pi \mathrm{a}\end{array}$ & $e_{u}$ & $\begin{array}{c}\sigma_{u}, \\
\text { МПа }\end{array}$ & $\begin{array}{c}\sigma_{Z} \\
\text { МПа }\end{array}$ & $\begin{array}{c}\sigma_{X} \\
\text { МПа }\end{array}$ & $\begin{array}{c}\sigma_{0}, \\
\text { МПа }\end{array}$ & $\eta$ \\
\hline \multirow{8}{*}{$\begin{array}{c}\mathrm{h}=1 \\
\mathrm{t}=9,5 \\
\mathrm{~b}=3,4 \\
\mathrm{r}=1,7 \\
\mathrm{x}_{1}=0,57 \\
\mathrm{x}_{2}=1,13 \\
\mathrm{x}_{3}=1,7\end{array}$} & 1 & 2440 & 0,72 & 840 & $-791,08$ & 48,92 & $-371,08$ & $-1,325$ \\
\hline & 2 & 2400 & 0,62 & 825 & $-776,96$ & 48,04 & $-364,46$ & $-1,325$ \\
\hline & 3 & 2420 & 0,675 & 835 & $-802,35$ & 32,65 & $-384,85$ & $-1,383$ \\
\hline & 4 & 2480 & 0,81 & 860 & $-842,87$ & 17,13 & $-412,87$ & $-1,44$ \\
\hline & 5 & 2430 & 0,72 & 840 & -840 & 0 & -420 & $-1,5$ \\
\hline & 6 & 2460 & 0,765 & 850 & -855 & 0 & $-427,5$ & $-1,5$ \\
\hline & 7 & 2440 & 0,72 & 840 & $-823,27$ & 16,73 & $-403,27$ & $-1,44$ \\
\hline & 8 & 2465 & 0,755 & 850 & $-833,07$ & 16,93 & $-408,07$ & $-1,44$ \\
\hline \multicolumn{9}{|c|}{2 этап } \\
\hline $\begin{array}{c}\rho, \\
\text { мм }\end{array}$ & $\begin{array}{l}\text { HV, } \\
\text { МПа }\end{array}$ & $\begin{array}{l}\sigma_{u}, \\
\text { МПа }\end{array}$ & $e_{u}$ & $\begin{array}{c}\theta, \\
\text { град }\end{array}$ & $\begin{array}{l}\sigma_{\rho} \\
\text { МПа }\end{array}$ & $\begin{array}{c}\sigma_{\theta}, \\
\text { МПа }\end{array}$ & $\begin{array}{l}\tau_{\rho \theta}, \\
\text { МПа }\end{array}$ & $\eta$ \\
\hline \multirow[t]{5}{*}{12,85} & 2400 & 825 & 0,573 & 0 & 85,4 & $-867,23$ & $\begin{array}{c}-17,56 \\
* 10^{-7} \approx 0\end{array}$ & $-1,422$ \\
\hline & & & & 5 & 85,97 & $-866,1$ & 16,33 & $-1,418$ \\
\hline & & & & 15 & 90,55 & $-856,94$ & 49,38 & $-1,393$ \\
\hline & & & & 25 & 99,72 & $-838,1$ & 83,67 & $-1,343$ \\
\hline & & & & 30 & 106,02 & $-824,7$ & 101,57 & $-1,307$ \\
\hline
\end{tabular}

Результаты расчетов напряжений в наиболее характерных точках и областях на примере схемы № 1, заготовка 1.1

В таблице 2 приведены результаты расчетов напряженного состояния и показателя $\eta$ для опасных с точки зрения разрушения областей для 5 заготовок. 
Таблица 2

Результаты расчета напряженного состояния и показателя $\eta$ для опасных с точки зрения разрушения областей для 5 заготовок

\begin{tabular}{|c|c|c|c|c|c|c|c|c|}
\hline \multicolumn{9}{|c|}{1 этап } \\
\hline № Заготовки & $\begin{array}{c}\text { № } \\
\text { точки }\end{array}$ & $\begin{array}{l}\mathrm{HV}, \\
\mathrm{MПа}\end{array}$ & $e_{u}$ & $\begin{array}{c}\sigma_{u} \\
\text { МПа }\end{array}$ & $\begin{array}{c}\sigma_{Z} \\
\mathrm{M \Pi а}\end{array}$ & $\begin{array}{c}\sigma_{X} \\
\text { МПа }\end{array}$ & $\begin{array}{c}\sigma_{0}, \\
\mathrm{M \Pi а}\end{array}$ & $\eta$ \\
\hline 1.1 & 4 & 2480 & 0,81 & 860 & $-842,87$ & 17,13 & $-412,87$ & $-1,44$ \\
\hline 1.2 & 5 & 2405 & 0,65 & 830 & -830 & 0 & -415 & $-1,5$ \\
\hline 2.1 & 2 & 2560 & 1,01 & 900 & $-856,11$ & 43,89 & $-406,11$ & $-1,354$ \\
\hline 2.2 & 5 & 2580 & 1,065 & 905 & -905 & 0 & $-452,5-$ & $-1,5$ \\
\hline 2.3 & 2 & 2420 & 0,675 & 835 & $-805,83$ & 29,17 & $-388,33$ & $-1,395$ \\
\hline \multicolumn{9}{|c|}{2 этап } \\
\hline$\rho$, мМ & $\begin{array}{l}\text { HV, } \\
\text { МПа }\end{array}$ & $\begin{array}{c}\sigma_{u} \\
\mathrm{M \Pi а}\end{array}$ & $e_{u}$ & $\begin{array}{c}\theta, \\
\text { град }\end{array}$ & $\begin{array}{l}\sigma_{\rho} \\
\text { МПа }\end{array}$ & $\begin{array}{c}\sigma_{\theta} \\
\text { МПа }\end{array}$ & $\begin{array}{l}\tau_{\rho \theta}, \\
\text { МПа }\end{array}$ & $\eta$ \\
\hline 12,85 & 2400 & 825 & 0,573 & 25 & 99,72 & $-838,1$ & 83,67 & $-1,343$ \\
\hline 12,35 & 2410 & 830 & 0,589 & 30 & 139,23 & $-797,72$ & 100,81 & $-1,19$ \\
\hline 12,25 & 2420 & 835 & 0,606 & 5 & 166,02 & $-797,62$ & 16,01 & $-1,135$ \\
\hline 12,1 & 2580 & 905 & 0,881 & 30 & 204,3 & $-817,36$ & 109,81 & $-1,016$ \\
\hline 9,65 & 2180 & 715 & 0,294 & 5 & 96,79 & $-728,39$ & 13,21 & $-1,325$ \\
\hline
\end{tabular}

На рис. 7 показана диаграмма пластичности стали 20X и область НДС в процессе формирования внутренних шлицевых поверхностей.

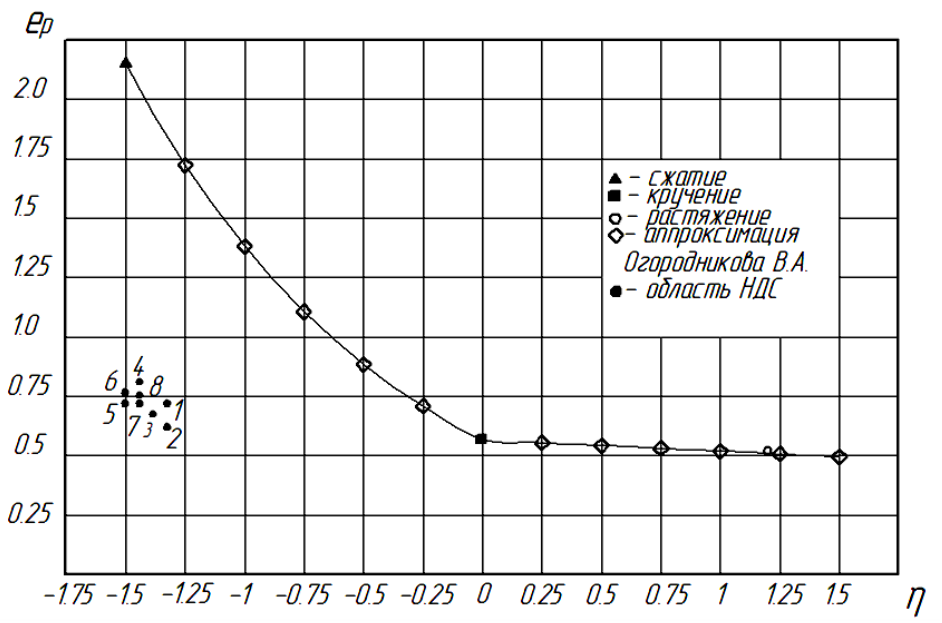

Рис. 7. Диаграмма пластичности стали $20 \mathrm{X}$ и область НДС

Использованный ресурс пластичности рассчитывали по критерию, предложенному Г. А. Смирновым-Аляевым (влиянием истории деформирования пренебрегается):

$$
\psi=\frac{e_{u}}{e_{p}(\eta)} \leq 1,
$$

$e_{p}(\eta)$ - предельная деформация в момент появления первых трещин, обнаруживаемых визуально; $e_{u}-$ уровень достигнутых деформаций.

В таблице 3 приведены результаты расчетов использованного ресурса пластичности для опасных с точки зрения разрушения областей для 5 заготовок. 
Результаты расчетов использованного ресурса пластичности

\begin{tabular}{|c|c|c|c|c|c|}
\hline № заготовки & 1.1 & 1.2 & 2.1 & 2.2 & 2.3 \\
\hline № точки & 4 & 5 & 2 & 5 & 2 \\
\hline$\psi$ & 0,4 & 0,3 & 0,53 & 0,5 & 0,34 \\
\hline
\end{tabular}

Оценка деформируемости заготовок в процессах обработки давлением базируется на феноменологической теории деформируемости, преимущество которой перед другими теориями разрушения заключается в том, что она учитывает влияние истории нагружения на интенсивность накопления повреждений. Теория деформируемости позволяет также получить количественную оценку влияния параметров технологического процесса на вероятность разрушения и, на основе этого, рационально использовать резервы пластичности.

В основе современной теории деформируемости лежит гипотеза о зависимости пластичности от схемы напряженного состояния, которая описывается диаграммой пластичности. Диаграммы пластичности в большинстве теорий описывают экспериментальную зависимость предельной деформации от показателя жесткости напряженного состояния. Однако в последних работах В. А. Огородникова, А. А. Богатова, Ю. Г. Важенцева, С. В. Смирнова показано, что зависимость пластичности от схемы напряженного состояния характеризуется, как минимум, двумя показателями напряженного состояния. В.А. Огородников считает, что при объемном напряженном состоянии необходимо учитывать влияние на пластичность третьего инварианта тензора напряжений. Такой подход позволил получить положительные результаты при разработке ряда процессов обработки давлением, которые реализуются в условиях объемного напряженного состояния.

Для оценки деформируемости заготовок и для технологического обеспечения качества готовых изделий необходимая информация об истории деформирования каждой материальной частицы в объеме заготовки. Достоверную информацию об истории деформирования можно получить только после решения краевой задачи теории пластичности.

\section{ВЫВОДЫ}

Рассмотрен перспективный процесс изготовления внутренних шлицевых поверхностей в глухих отверстиях методом ХПД.

Показана целесообразность изучения механики процесса формирования внутренних шлицевых поверхностей в глухих отверстиях.

Предложены различные схемы формирования внутренних шлицевых поверхностей в глухих отверстиях с целью определения наиболее благоприятной схемы с точки зрения деформирования заготовки без разрушения.

Изучено НДС в процессе формирования внутренних шлицевых поверхностей в глухих отверстиях методом холодного пластического деформирования.

Оценен ресурс пластичности заготовок из стали $20 \mathrm{X}$ с помощью феноменологического критерия деформируемости. Показано, что в рассматриваемом процессе ресурс пластичности заготовок составил $0,53 \geq \psi \geq 0,3$.

Наиболее благоприятной, с точки зрения использованного ресурса пластичности, является комбинированная схема нагружения (заготовка № $1.2, \psi=0,3$ ).

\section{СПИСОК ИСПОЛЬЗОВАНОЙ ЛИТЕРАТУРЫ}

1. Данилов В. А., Данилов А. А. Анализ и реализация методов управления макрогеометрией сложных многогранных поверхностей при обработке резанием. Вестник Полоцкого государственного университета. Серия В. Промышленность. 2015. 11. С. 2-9.

2. Амбросимов С. К., Грибков Р. В. Деформирующе-режущее протягивание со смещением режущих кромок относительно поверхности резания. Вестник Липецкого государственного технического университета. 2019. 2 (40). C. 41-46.

3. Кацев П. Г. Обработка протягиванием. Справочник (Серия справочников для рабочих). Москва: Машиностроение, 1986. 272 с. 
4. Данилов В. А., Данилов А. А. Управление топологией некруглых поверхностей, обработанных по схемам неполного формообразования. Вестник Полоцкого государственного университета. Серия В. Промышленность. 2015. 4. С. 2-9.

5. Олифиренко М. И. Прогрессивные процессы зубодолбления. Киев: Техника, 1988. 190 с.

6. Атрошенко А. П., Федоров В. И. Горячая штамповка труднодеформируемых материалов. Ленинград: Машиностроение, 1979. 287 с.

7. Розенберг О. А., Траченко Б. П. Формообразования шлицевых отверстий в трубных заготовках методом холодного пластического деформирования. Технология и организация производства. 1991. 2. С. 20-23.

8. Шейкін С. С., Студенець С. Ф., Мельниченко В. В., Мельниченко Я. В. Технологія відновлення карданних валів з застосуванням градієнтного деформаційного зміцнення. Високі технологї в машинобудуванні. Харків: НТУ «ХПІ». 2016. 1 (26). С. 118-125.

9. Розенберг О. А., Студенец С. Ф., Мельниченко В. В., Кирица И. Ю. Деформируемость металла при формировании внутренних шлицевых поверхностей в глухих отверстиях методом холодного пластического деформирования (Сообщение 1). Вісник Донбаської державної машинобудівної академії. Краматорськ. 2007. 1(7). С. $117-120$.

10. Огородников В. А., Савчинский И. Г, Нахайчук О. В. Напряженно - деформированное состояние при формировании внутреннего шлицевого профиля методом обжатия на оправке. Тяжелое машиностроения. 2004. 12. С. $31-33$

11. Огородніков В. А., Кириця І. Ю., Перлов В. С. Механіка процесів холодного пластичного деформування вісесиметричніх заготовок з глухим отвором: монографія. Вінниця: ВНТУ, 2015. 164 с.

12. Кириця І. Ю. Особливості розрахунку використаного ресурсу пластичності при холодному формуванні внутрішніх шліцьових поверхонь в глухих отворах. Вісник ХНУ. Хмельницький. 2015. 5. С. 119-122.

\section{REFERENCES}

1. Danilov V.A, Danilov A.A. Analysis and implementation of methods for controlling the macrogeometry of complex polyhedral surfaces during cutting. Bulletin of Polotsk State University. Series B. Industry. 2015. 11, pp. $2-9$. (in Russian).

2. Ambrosimov S.K., Gribkov R.V. Deforming-cutting broaching with displacement of cutting edges relative to the cutting surface. Bulletin of Lipetsk State Technical University. 2019. 2 (40), pp. 41-46. (in Russian).

3. Katsev P.G. Broaching processing. Handbook. Moscow: Mechanical Engineering. 1986. 272 p. (in Russian).

4. Danilov V.A., Danilov A.A. Topology control of non-circular surfaces processed according to incomplete shaping schemes. Bulletin of Polotsk State University. Series B. Industry. 2015. 4, pp. 2-9. (in Russian).

5. Olifirenko M.I. Progressive gear shaping processes. Kiev: Technique. 1988. 190 p. (in Russian).

6. Atroshenko A.P., Fedorov V.I. Hot stamping of hard-to-form materials. Leningrad: Mechanical Engineering. 1979. 287 p. (in Russian).

7. Rosenberg O.A., Trachenko B.P. Forming of spline holes in tubular blanks by cold plastic deformation. Technology and organization of production. 1991. 2, pp. 20-23. (in Russian).

8. Sheikin S.E., Studenets S.F., Melnichenko V.V., Melnichenko J.V. Technology of restoration of cardan shafts with application of gradient deformation strengthening. High technologies in mechanical engineering. Kharkiv: NTU "KhPI". 2016. 1 (26), pp. 118-125. (in Ukrainian).

9. Rosenberg O.A., Studenets S.F., Melnichenko V.V., Kyrytsia I.Yu. Deformability of metal during the formation of internal spline surfaces in blind holes by cold plastic deformation (Communication 1). Herald of DSEA. Kramatorsk 2007. 1 (7), pp. 117-120. (in Russian).

10. Ogorodnikov V.A., Savchinsky I.G., Nakhaichuk O.V. Stress - deformed state during the formation of an internal spline profile by the method of compression on a mandrel. Heavy engineering. 2004. 12, pp. 31-33. (in Russian)..

11. Ogorodnikov V.A., Kyrytsia I.Yu., Perlov V.E. Mechanics of cold plastic deformation of axisymmetric workpieces with a blind opening: monograph. Vinnitsa: VNTU. 2015. 164 p. (in Ukrainian).

12. Kyrytsia I.Yu. Features of calculation of the used resource of plasticity at cold formation of internal slotted surfaces in blind apertures. Bulletin of KhNU. Khmelnytskyi. 2015. 5, pp. 119-122. (in Ukrainian).

Огородников В. А. - д-р техн. наук, проф. ВНТУ;

Кирица И. Ю. $\quad$ - канд. техн. наук, доц. ВНТУ.

ВНТУ - Винницкий национальный технический университет, г. Винница.

E-mail: slk-vin@ukr.net

Статья поступила в редакциию 15.01.2020 ح. 